\title{
Proyecto de apropiación de las TIC en servicios sociales de atención social primera del Ayuntamiento de Madrid
}

\author{
Project to appropriation ICTs in the primary social care services provided \\ by Madrid City Council
}

\author{
José Ignacio SANTÁs GARCÍA \\ Ayuntamiento de Madrid, España \\ santasgji@madrid.es
}

Recibido: 03/02/2016

Revisado: 02/03/2016

Aceptado: 06/06/2016

Disponible on line: 01/07/2016

\begin{abstract}
Resumen
La implementación de las Tecnologías de la Información y Comunicación en el campo de los Servicios Sociales implica un enorme espectro de oportunidades para la visibilidad, la eficiencia y la gestión interna, a la vez que posibilita una apertura en cuanto al acceso a los mismos por parte de la ciudadanía. Además, las múltiples oportunidades que las TIC suponen para la inclusión social exigen que los Servicios Sociales y el Trabajo Social se apropien de las mismas como herramienta básica en la Intervención Social.
\end{abstract}

Palabras clave: tecnologías de la información y la comunicación, servicios sociales, Trabajo Social, atención social primaria.

\begin{abstract}
The incorporation of ICTs within the field of social services implies an enormous spectrum of opportunities for visibility, efficiency and internal management, at the same time as it provides an opportunity to open them up to citizen access. Moreover, the multiple opportunities that ICTs offer for social inclusion demands that social services and social work make use of them as a basic tool in social intervention.
\end{abstract}

Keywords: ICTs, social services, social Work, primary social care.

Referencia normalizada: Santás García, J. I. (2016): «Proyecto de apropiación de las TIC en servicios sociales de atención social primaria del Ayuntamiento de Madrid». Cuadernos de Trabajo Social, 29(2): 213-223.

Sumario: Introducción. La sociedad de la información y el conocimiento y el Trabajo Social. 1. Uso de las Tecnologías de la información y la comunicación: sistemas de tratamiento de la información en la atención social a través de los Servicios Sociales. 2. Contribución de las Tecnologías de la Información y la Comunicación a la intervención social y la exclusión social. 3. Proyecto de aplicación de las Tecnologías de la Información y la Comunicación a los Servicios Sociales. 4. Conclusiones. 5. Referencias bibliográficas.

Introducción. La sociedad de la información y el conocimiento y el Trabajo Social

Nuestra sociedad se encuentra en una verdadera revolución, inmersa en lo que se viene denominando desde la década de 1980 (González-Seara, 1981) como Sociedad de la Información y el Conocimiento (SIC), término que se emplea para diferenciarla de su antecesora: la sociedad postindustrial. A 35 años de entonces, podemos decir que no sólo está caracterizada por el nacimiento de un sector económico «cuaternario» (relativo al tratamiento de la información) ni es meramente una revolución tecnológica, sino económica, cultural y social global.

De hecho, el impacto de las Tecnologías de la Información y el Conocimiento en la transmisión y la gestión de la información, como indica Castells (2007), se compara con la invención de la imprenta en la Edad Media. Si ésta supuso una ruptura con las restricciones del entorno eclesiástico al acceso y procesamiento del conocimiento, gracias a estas tecnologías (TIC), el conocimiento, procesamiento y transmisión de la información se encuentran para la 
ciudadanía, en la actualidad, en acceso prácticamente libre.

Siguiendo las aportaciones de Grovi (2007), se podrían establecer tres niveles en la incorporación de las TIC por las instituciones:

- Acceso: se refiere al modo de acercarse a algo con el propósito de conseguir su dominio. El nivel de acceso se refiere únicamente a la posesión física de la tecnología. Acceso y dominio son dos términos que marcan una diferencia evidente que, a su vez, define el alcance de las políticas de impulso de las TIC, al igual que no es lo mismo proveer de infraestructura técnica que ayudar a dominarla. Se realiza esta distinción ante la experiencia constatada de que el impulso no puede quedarse en la simple adquisición de equipos, que - lejos de un uso - quedan obsoletos en poco tiempo, y por lo tanto, la inversión no estará amortizada si la capacitación de las personas a las que se dirige la iniciativa ha sido insuficiente.

- Uso: derivado de la preocupación por el acceso, se habla frecuentemente del concepto de uso de las TIC, entendiéndolo como el ejercicio o la práctica habitual y continuada de un artefacto tecnológico (si bien no indica el para qué se usa). Aunque no existen límites claros, se puede hablar de tres niveles de capacitación digital:

- Básico (habilidad para manejar programas de uso general).

- Intermedio (habilidad para manejar programas de mayor complejidad).

- Avanzado (habilidad para el manejo de programas más sofisticados en su forma y otros más avanzados, como software de gestión, manejo de redes sociales, etc.).

- Apropiación: los procesos de apropiación implican el dominio del objeto cultural, pero también el reconocimiento de la actividad que condensa ese instrumento y, con ella, los sistemas de motivaciones, el sentido cultural del conjunto. La apropiación se produce por la participación de hecho en la actividad que se lleva a cabo con las TIC, que comienza de manera gradual (incluso asistida inicialmente), pero luego el usuario encuentra caminos propios e independientes.

Así, las TIC no sólo han revolucionado nuestros contextos más próximos (domésticos y fa- miliares), sino también los laborales y sectores más amplios como la Economía, la Política, la Sanidad y los Servicios Sociales, entre otros muchos: su omnipresencia es tal que ya no es posible concebir ninguna disciplina sin la implementación de las TIC ni existe posibilidad de volver al escenario anterior.

A su vez, el uso de las TIC se ha convertido en un elemento diferenciador capaz de aumentar el capital relacional de las personas, concepto vinculado directamente con el de inclusión social y, como no puede ser de otra forma, con la disciplina del Trabajo Social, que «promueve el cambio y el desarrollo social, la cohesión social y el fortalecimiento y liberación de las personas»» (FITS, 2015). Por ello, no podemos situarnos ni quedar al margen de esta realidad, ya que las nuevas tecnologías se convierten en un elemento fundamental para poder alcanzar los objetivos que nos definen como profesión; el capacitarnos en su utilización, en su conocimiento y en sus posibilidades debe significar un reto para todas $\mathrm{y}$ todos los/as trabajadores/as sociales porque brindan magníficas oportunidades para el desarrollo del conjunto de nuestras funciones (Arredondo Castellano y Celimendiz, 2012).

Lamentablemente, en los contextos institucionales relacionados con la intervención social, la incorporación de las nuevas tecnologías y su desarrollo es aún medio (Cabrera et al., 2005, p. 163) debido a varios factores como: la carencia de medios económicos, técnicos y formativos principalmente. Todo ello a pesar de la ganancia neta que suponen las TIC, no sólo para los colectivos con los que trabajan los trabajadores y las trabajadoras sociales, sino para el propio desarrollo de la acción profesional: especialmente, en la eficiencia, difusión, impacto, ahorro de costes (economía, ecología y rapidez), participación, transparencia y, por lo tanto, en democracia.

\section{Uso de las Tecnologías de la Información y la Comunicación: sistemas de tratamiento de la información en la atención social a través de los Servicios Sociales}

La implantación de las TIC en los Servicios Sociales está unida a los sistemas de tratamiento de la información aplicados y su desarrollo es el fiel reflejo de la situación de este sistema de protección en España, caracterizada por su fragmentación. Esta fragmentación nace desde la creación 
de los Servicios Sociales en los años 1980, dado que las competencias en Asistencia Social (ahora Servicios Sociales) corresponden a las Comunidades Autónomas, desde la promulgación de la Constitución Española de 1978 (Artículo 148.20). A partir de entonces, cada Comunidad Autónoma ha desarrollado sistemas de Servicios Sociales con ciertas similitudes y diferencias, incrementándose estas últimas cuanto mayor es la atomización por municipios o territorios concretos, dado que se prestan por las comunidades autónomas, diputaciones o corporaciones locales, según la Ley Reguladora de Bases de Régimen Local (7/85, artículo 25.2. k), que establece que los municipios con más de 20.000 habitantes (Artículo 26 c) serán quienes presten los Servicios Sociales a sus vecinos y vecinas.

Ante esta complejidad estructural y por la inexistencia de una ley de carácter estatal, se impulsó la homogeneización, a través del Acuerdo de 1988 entre el entonces Ministerio de Trabajo y Asuntos Sociales y las Comunidades Autónomas, en el que nació el Plan Concertado para las Prestaciones Básicas de Servicios Sociales en las corporaciones locales, que estableció ciertos criterios comunes gracias a un sistema de cofinanciación de estas prestaciones, según se recogía en el citado acuerdo.

Por lo tanto, para poder hablar de las TIC en Servicios Sociales públicos, es necesario una mención especial a los sistemas de tratamiento de la información que emplean en el desarrollo de la atención social.

Fruto del Plan Concertado fue la creación en 1994 del Sistema de Información de Usuarios de Servicios Sociales (SIUSS), desarrollado por el actual Ministerio de Sanidad, Servicios Sociales e Igualdad, en colaboración con las Comunidades Autónomas.

Así, el SIUSS, en el nivel de toma de datos -que corresponde a los Servicios Sociales municipales, a través de la Unidad de Trabajo Social (UTS) - se estructura en cuatro niveles (IMSERSO, 2011):

- Unidad de Trabajo Social (UTS): en este nivel se encuentra el contenido y desarrollo de la ficha social. Está diseñado para su utilización por el trabajador o la trabajadora social y, en el conjunto del programa, es el nivel de toma de datos.

- Centro de Servicios Sociales: recibe los datos de la UTS para su propia gestión y explo- tación. Define la UTS y sus zonas básicas de atención.

- Comunidad Autónoma: recibe los datos del Centro de Servicios Sociales para sus propias explotaciones. Define la estructura de los centros, la zonificación territorial de la atención y los sectores de población atendida.

- Ministerio: recibe los datos de las Comunidades Autónomas y realiza las explotaciones de todo el territorio.

Este programa, instalado en las Comunidades Autónomas, Centros de Servicios Sociales y UTS, permite obtener, mediante el tratamiento de datos y las explotaciones estadísticas correspondientes, los siguientes resultados: sociales

- Perfil de usuarios y usuarias de servicios

- Perfil de usuarios y usuarias de cada una de las prestaciones básicas.

- Información sobre las demandas y su evolución temporal.

- Información sobre las intervenciones profesionales y recursos aplicados

Así pues, esta aplicación se configuró mediante expedientes familiares y permite a los trabajadores y a las trabajadoras sociales de base su gestión, según los criterios establecidos por el propio Ministerio de Sanidad, Servicios Sociales e Igualdad.

No obstante, esta aplicación informática no ha estado exenta de cierta crítica por el Trabajo Social que la acusa de producir cierto encajamiento de la disciplina en la simple gestión de la prestación de servicios y recursos. La estructura de la intervención del SIUSS puede ser uno de los más claros (y extendidos) ejemplos de un modelo de actuación basado en el binomio Demanda-Recurso, esto es, ante una demanda, la intervención se justifica con la aplicación de un recurso. Este aspecto es desde hace años cuestionado y denunciado en los diferentes foros profesionales, y existe una opinión generalizada que considera que es uno de los factores que más alejó a la profesión de sus funciones esenciales $(\mathrm{Pu}-$ ñal, 2004).

Es más, en ocasiones se ha atribuido al Sistema de Información de Usuarios de Servicios Sociales cierta responsabilidad en la burocratización de la propia intervención social: «Generó 
una forma de intervención burocrática y administrativa, que absorbió y obstaculizó las intervenciones psico-sociales y la aplicación del cuerpo teórico-técnico (Gil et al., 1996, citado en Puñal, 2004).

Dejando de lado esta crítica, que aporta una importante reflexión sobre el trabajo cotidiano en la atención a la ciudadanía, se describen a continuación las utilidades del SIUSS, que pueden agruparse en dos grandes bloques: Gestión y Explotación de los datos.

1. Gestión de prestaciones y expedientes:

- Gestión de prestaciones.

- Búsqueda de información.

- Facilita el seguimiento de actuaciones:

- Consulta.

- Obtención rápida de datos e informes.

- Sirve de apoyo a un diagnóstico social adecuado.

- Posibilita el almacenamiento y organización de la información.

2. Explotación de los datos almacenados y utilidades estadísticas:

- Directa: se obtienen estadísticas prediseñadas en los cuatro niveles de instalación (Unidades de Trabajo Social, Centros de Servicios Sociales, Comunidades Autónomas y Ministerio).

- Exportación de datos a paquetes estadísticos más complejos.

— Estadísticas.

En cuanto a los resultados de uso de esta herramienta, el SIUSS permite, a cada una de las administraciones, dentro de su ámbito territorial, realizar el tratamiento de los datos y las explotaciones estadísticas correspondientes, obteniendo información sobre los siguientes resultados:

- Perfil de los usuarios de servicios sociales.

- Perfil de usuarios de cada una de las prestaciones básicas.

- Información sobre las demandas y su evolución temporal.

- Información sobre las intervenciones profesionales y recursos aplicados.
De esta forma, tanto las UTS como los Centros de Servicios Sociales y las Comunidades y Ciudades Autónomas pueden obtener información sobre las demandas y los recursos, hasta el nivel de desagregación definido por la Comunidad Autónoma.

Como consecuencia de la fragmentación descrita, no todos los municipios utilizan SIUSS. Cada vez es mayor el número de ellos que crean sus propias aplicaciones informáticas, como es el caso del Ayuntamiento de Madrid.

Indudablemente, dadas las posibilidades actuales que las TIC pueden ofrecer para la gestión de la atención social, y al margen de las utilidades de SIUSS, hoy por hoy cualquier aplicación informática de Servicios Sociales debería permitir la gestión integral, incluyendo, al menos, las siguientes funciones:

- Agenda y cita previa: agenda propia para cada profesional, que la pueda programar; la gestión centralizada de las agendas de cada centro, tanto para la atención individual como grupal, así como la solicitud de cita previa en línea

- Soportes documentales, formatos de recogida y gestión de toda la información social: ficha, intervención social - entrevistas, visitas a domicilio, coordinaciones-, historia social, etc.; así como la explotación sencilla y la elaboración de informes estadísticos, teniendo en cuenta siempre la compatibilidad entre aplicaciones.

- Formatos de interacción y coordinación interadministrativa (derivación, formularios, etc.) y con entidades colaboradoras por parte de los y las profesionales.

- Gestión de las prestaciones sociales municipales, autonómicas y estatales pertinentes.

- Gestión y registro de actividades por sectores de población y colectivos específicos (familia, mujer, dependencia, etc.); así como filtro de perfiles, según características concretas, con la finalidad de poder ofrecerles atenciones adaptadas a su tipología.

- Programación e intervención grupal y comunitaria.

- Realización de trámites y consultas por la ciudadanía de manera telemática.

- Contemplar módulos de relación entre usuarios y profesionales, así como la gestión del flujo de información bidireccional (lo incluye última tendencia). 
Todo ello, unido a la necesaria aplicación de la Ley de acceso electrónico de los ciudadanos a los servicios públicos (Ley 11/2007 de 22 de junio), que establece el derecho a realizar tramitaciones o consultas de manera digital.

Por otro lado, la carga de trabajo de los profesionales del campo social y la presión asistencial exigen incorporar sistemas de eficiencia que sólo pueden implementarse con la incorporación de las TIC al ámbito organizacional, facilitando: la apropiación de las TIC, la planificación, la reducción de esperas, la organización de desplazamientos, y la posibilidad de realizar una intervención social intensiva, al disminuir el número de cargas y tareas administrativas.

Por supuesto: la aplicación de las TIC a este campo no sustituye el contacto presencial, sino que refuerza la creación de tiempos con calidad, dado que conlleva la eliminación de gestiones y contactos innecesarios pudiendo centrar la relación en lo esencial: la intervención social. Del mismo modo, reduce los tiempos de gestión dedicados a tareas de índole burocrática, y, por lo tanto, favorece la aparición de un vínculo significativo, condición necesaria para desarrollar una intervención profesional adecuada.

\section{Contribución de las Tecnologías de la In- formación y la Comunicación a la interven- ción social y la exclusión social}

La exclusión social se define (Cabrera et al, 2005) como:

Un proceso dinámico y cambiante que cursa con una quiebra de la identidad personal y que normalmente hunde sus raíces en un debilitamiento, mayor o menor, de los dos ejes básicos en los que se fundamenta la inserción social y compuesta por dos ejes:

a) socio-relacional merced al cual se dispone de una serie de apoyos, vínculos sociales y contactos, con familiares, amigos, vecinos, colegas..., que constituyen nuestro capital relacional.

b) económico-laboral, habitualmente merced a un empleo por el que se recibe un salario que se constituye como el componente fundamental de capital económico, base material para afrontar las contingencias de la existencia (p. 13).

Castells (2007) señala que los usuarios más activos y frecuentes de Internet, cuando se comparan con los no usuarios, son personas más sociables, tienen más amigos, más intensidad de relaciones familiares, más iniciativa profesional, menos tendencia a la depresión y al aislamiento, más autonomía personal, más riqueza comunicativa y más participación ciudadana y sociopolítica.

Resulta, pues, evidente que un adecuado uso de las TIC puede ampliar enormemente el capital relacional y, en consecuencia, los beneficios en la dimensión social y laboral, de manera que pueden contribuir a evitar situaciones de exclusión social, así como a utilizar los recursos adecuados para poder salir de las mismas.

La exclusión de estas oportunidades, unido al avance cada vez más tecnológico de la sociedad, descubren un nuevo peligro: las TIC «pueden alejar» a las personas en situación de exclusión social si no se facilitan los medios y canales para su uso a este segmento de la población.

Nuestro código deontológico señala en su artículo 17 que los/las profesionales del Trabajo Social se comprometen en la intervención social a buscar y garantizar a toda persona, grupo o comunidad la igualdad de oportunidades, el acceso a los recursos y el apoyo para cubrir sus necesidades; especialmente de aquellos que se encuentran en situación de mayor vulnerabilidad o en alguna situación específica de desventaja social.

Por ello, la intervención social, especialmente desde los espacios con responsabilidad pública en el bienestar social, como es el de los Servicios Sociales, debe contemplar la oferta de acciones de capacitación tecnológica para prevenir que ciertos sectores vulnerables -o que ya viven las diferentes formas de exclusión- queden invisibilizados o «infoexcluidos» como lo denominan algunos autores (Colás, 2004). Ejemplos de utilidades los hay múltiples: desde la búsqueda de empleo (cuyo método tradicional de entrega de curriculum presencial está en declive) hasta la gestión documental que, realizada presencialmente, comporta importantes costes económicos, especialmente para quien carece de ellos.

Si bien es preciso utilizarlas de manera general en el campo de la intervención social, pueden señalarse diversas situaciones específicas para las cuales las TIC son especialmente recomendables (Arriazu y Fernández-Pacheco, 2013, p. 156), en concreto cuando existen:

- Inconvenientes en el proceso comunicativo, por ejemplo, discapacidad auditiva o barreras lingüísticas. 
- Problemas de movilidad o determinados trastornos que impiden realizar desplazamientos.

- Problemáticas geotemporales. La conciliación laboral y familiar, en ocasiones, encuentra en los procesos de intervención dificultades que podrían resolverse mediante el uso de las TIC.

- Situaciones de riesgo: en ocasiones, la intervención individual presencial supone riesgos que pueden evitarse mediante el uso de las TIC.

Estos autores (idem) además, señalan a su vez ventajas e inconvenientes de las TIC, que son anotadas en el Cuadro 1.

De hecho, buena parte de los argumentos que se han esgrimido para situarse en contra de la práctica en línea del trabajo social no tienen sentido, han dejado de tenerlo o son fáciles de rebatir (Arias Astray, Alonso Gónzález y Corchado Castillo, 2013):

- Por ejemplo, la fiabilidad de la tecnología está mejorando de manera muy sustantiva, por lo que son cada vez menos relevantes los reparos tradicionales manifestados en este sentido.

- Los problemas de falsa identidad tienen fácil solución mediante la identificación con las huellas digitales o el escaneo de las pupilas, por ejemplo.
- La riqueza y calidez de la comunicación en línea mejora cada día y lo seguirá haciendo cada vez más, en la medida en que se vaya optimizando el ancho de banda.

- La facilidad de uso, la portabilidad, el precio, y la relación entre costes y beneficios del uso de las TIC en Trabajo Social mejoran a diario.

- Las TIC han mostrado tener mayor poder de inclusión que de exclusión, como inicial y erróneamente se pensaba.

\section{Proyecto de aplicación de las Tecnologías de la información y la comunicación a los Ser- vicios Sociales}

Según la Carta de Servicios de los Centros de Servicios Sociales de Atención Social Primaria del Ayuntamiento de Madrid, éstos constituyen el primer nivel de atención del Sistema Público de Servicios Sociales, y son la instancia más próxima para la atención social a la ciudadanía. Esta atención se lleva a cabo en los Centros de Servicios Sociales, que constituyen la puerta de acceso a la atención social y a todos los servicios y prestaciones sociales.

Por lo tanto, el interés del siguiente proyecto estriba en la apropiación de las TIC —el significado de su acceso y uso se ha explicado ya- por los Servicios Sociales municipales públicos, no

\begin{tabular}{|c|c|}
\hline VENTAJAS & INCONVENIENTES \\
\hline $\begin{array}{l}\text { - Flexibilidad, accesibilidad y fluidez en la rela- } \\
\text { ción entre profesional y usuario. } \\
\text { - Se logra un análisis más exhaustivo del com- } \\
\text { ponente verbal, muy valorado en distintos mode- } \\
\text { los y enfoques de intervención social. } \\
\text { - Facilidad en el registro y sistematización de la } \\
\text { información obtenida durante la todo el proceso } \\
\text { intervención social. } \\
\text { - Permite articular un modelo de intervención } \\
\text { claro y reflexivo donde el profesional y el usuario } \\
\text { cuenten con mayor margen temporal para la expli- } \\
\text { cación del problema y su posterior diagnóstico. } \\
\text { - Favorece el anonimato y la confidencialidad } \\
\text { del usuario a través de Internet. } \\
\text { - En las últimas fases de la intervención puede } \\
\text { utilizarse como vía para deshabituar la relación del } \\
\text { usuario con el profesional en la búsqueda de la au- } \\
\text { tonomía integral de la persona. }\end{array}$ & $\begin{array}{l}\text { - Problemas de acceso y cobertura técnica por } \\
\text { parte del profesional o persona demandante de } \\
\text { ayuda. } \\
\text { - Dificultad para establecer condiciones de em- } \\
\text { patía con el usuario que permitan profundizar en } \\
\text { los problemas latentes. } \\
\text { - Pérdida de la comunicación no verbal que di- } \\
\text { ficulta la comprensión global de los problemas del } \\
\text { usuario. } \\
\text { - Necesidad de conocer la normativa legal y ju- } \\
\text { rídica del contexto del usuario si la intervención } \\
\text { social se realiza en contextos geográficos diferen- } \\
\text { tes. } \\
\text { - Necesidad de formación especializada de pro- } \\
\text { fesionales en el manejo y uso de este tipo de herra- } \\
\text { mientas. }\end{array}$ \\
\hline
\end{tabular}

Cuadro 1. Ventajas e inconvenientes de las TIC.

Fuente: Arriazu, R. y Fernández-Pacheco, J.L. (2013). Internet en el ámbito del Trabajo Social: formas emergentes de participación e intervención socio-comunitaria. Cuadernos de Trabajo Social, 26(1): 149-158. 
sólo como herramienta de mejora de la eficiencia y la comunicación administrativa - que ya existe en la mayoría de administraciones públicas - ampliando el acceso de la ciudadanía al sistema, sino también de consecución de mayores cotas de autonomía e inclusión social de la misma.

\subsection{Inicio del proyecto}

El Concurso de Innovación «Innovando Juntos» fue una iniciativa de intraemprendimiento del Área de Gobierno de Economía, Hacienda y Administración Pública del Ayuntamiento de Madrid.

Este concurso se convocó en junio de 2014, bajo el siguiente reto: Aprovechar la experiencia y el conocimiento del personal municipal para la mejora de los servicios públicos y su aplicación práctica.

Los objetivos específicos eran:

- Promover la participación del personal,

- Crear sentimiento de pertenencia a la organización y

- Aprovechar el talento interno.

En el concurso podía participar la totalidad de la plantilla del Ayuntamiento de Madrid (más de 28.000 personas).

Este concurso tuvo las siguientes fases:

Fase 1: Aportación de ideas. Se presentaron un total de 287 ideas, de las que se seleccionaron $100 \mathrm{y}$, a su vez, pasaron a un jurado que redujo a 20 la ideas semifinalistas, mediante informes de las áreas implicadas en cada una de ellas.

Fase 2: Toda la plantilla tuvo un periodo para votar a esas 20 ideas, de ellas quedaron 5 finalistas: fue el caso del Proyecto de Acceso y Uso de los Servicios Sociales a través de las TIC, que es conocido como «Proyecto de aplicación de las TIC a los Servicios Sociales».

A continuación, se crearon grupos de trabajo: las personas que lideraban estos proyectos formaron equipos que llevaron a cabo la elaboración de los proyectos, en un periodo de un mes, recibiendo formación específica por los profesionales de distintas áreas, a iniciativa de la Oficina de Innovación Social del Ayuntamiento de Madrid.
El equipo que diseñó el Proyecto estaba formado por 5 profesionales de los Servicios Sociales municipales (Distritos y Área de Gobierno), Informática y Administración Electrónica del Ayuntamiento de Madrid.

Durante este periodo, se elaboró un dossier de estudio de viabilidad del proyecto, un análisis de pilotaje, un calendario de implementación, etc. Además, se usaron diversas técnicas de diseño de proyectos (como el CANVAS, BLUEPRINT, Costumer Journey Map, o el DAFO CAME) que ayudaron a la redacción final del proyecto.

Fase 3: Presentación de los proyectos ante un jurado en una sesión pública.

Posteriormente, se hizo público el reconocimiento del mejor proyecto. El Proyecto de Acceso y Uso de los Servicios Sociales a través de las TIC, que si bien ganó el concurso, obtuvo el compromiso de que sería desarrollado por la corporación municipal. Esta decisión trajo consigo el inicio de su implementación, en los primeros meses de 2015.

\subsection{Dimensiones del proyecto}

El proyecto se concibe con 3 dimensiones:

- El uso de las TIC para los profesionales, que podrán usar tablets para mejorar la eficiencia de las visitas domiciliarias y aumentar la calidad de las mismas.

- El uso de las TIC para personas usuarias, que podrán interaccionar con sus profesionales de referencia del Sistema de manera cómoda, inmediata y ágil, a la vez que recibir ofertas de empleo o información de interés, y mientras tanto pueden participar en talleres de capacitación tecnológica. Ello contribuirá a la disminución de los tiempos de espera para atención presencial.

- El uso de las TIC para visibilizar la labor que llevan a cabo los Servicios Sociales en las redes sociales.

\subsection{Descripción del proyecto}

El proyecto se titula: Acceso y uso de los Servicios Sociales a través de las TIC. (Proyecto de aplicación de las TIC a los Servicios Sociales).

Se diagnóstico se resume en:

- El aumento de la demanda actual y la previsión de incremento de cobertura exige mejorar la eficiencia de los Servicios Sociales. 
- El cambio de perfil tecnológico en la población requiere una actualización de los procesos de atención para agilizar la gestión de las prestaciones sociales a la ciudadanía.

- El desconocimiento de la oferta de prestaciones sociales conlleva de manera continua un pérdida de oportunidades para la ciudadanía.

Para llegar a ese diagnóstico, se realizaron las siguientes acciones:

- Focus group con usuarios de un Centro de Servicios Sociales

- 30 encuesta a trabajadores sociales.

- Redes sociales: sondeo en Facebook (perfil profesionales).

- Otras fuentes de información fueron:

- Sugerencias y Reclamaciones del Ayuntamiento de Madrid.

- Encuesta de satisfacción de usuarios de Servicios Sociales.

- Cartas de servicios del AG de Familia y Servicios Sociales.

- Observatorio de la Ciudad

- Fuentes bibliográficas y datos estadísticos

El reto era: Mejorar el acceso y la eficiencia de la Atención Social adaptándola a los nuevos contextos de la Ciudad de Madrid a través de las Tecnologías de la Información y la Comunicación

Los objetivos perseguidos:

- Facilitar el acceso y uso a los Servicios Sociales a la ciudadanía a través de las Tecnologías de la Información y la Comunicación (TIC).

- Acortar tiempos de espera para la atención social y tramitación de prestaciones sociales.

- Incrementar la visibilidad de los Servicios Sociales municipales de Atención Social Primaria.

La metodología se establece en función de cuatro líneas de acción:

Línea 1. Se modificará la aplicación informática empleada en los Servicios Sociales municipales del Ayuntamiento de Madrid: CIVIS. Esta aplicación, actualmente accesible únicamente por intranet, se convertirá en una plataforma,por la que las personas usuarias podrán: acceder a perfiles propios, consultar el estado de sus prestaciones, recibir información de interés según su perfil e interaccionar con su profesional de referencia. Esta línea es la fundamental y en donde realmente hay una mayor innovación de cara a la intervención social.

Línea 2. De igual forma, los Centros de Servicios Sociales ofrecerán talleres de capacitación tecnológica a las personas que lo necesiten y para ello seguirán protocolos de captación para perfiles predefinidos, derivándolas a los recursos disponibles para este fin. En estos talleres se priorizará el aprendizaje de uso de smartphone utilizando el wifi de los Centros de Mayores Municipales, de libre acceso.

Línea 3. Los trabajadores y las trabajadoras sociales dispondrán de tablets para las visitas a domicilio. Ello posibilitará la cumplimentación de los baremos de valoración funcional mediante los que se regula la intensidad de los servicios domiciliarios, además de otros baremos; asimismo se podrá citar, cumplimentar registros o consultar las historias sociales en línea desde el propio domicilio.

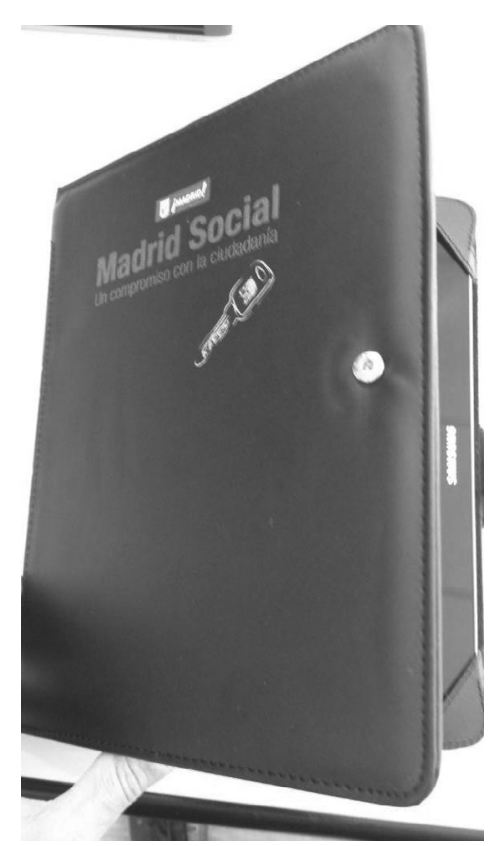

Figura 1. Tablet usada por los Centros de Servicios Sociales del Ayuntamiento de Madrid. 
Línea 4. La labor realizada por los Servicios Sociales la visualiza el Área de Gobierno, creando los cauces oportunos, dentro de la web corporativa, y los perfiles en las redes sociales municipales.

Los resultados esperados fueron los siguientes:

1. Proporcionar información clara y accesible de la oferta municipal de recursos y ayudas de los Servicios Sociales a través de la aplicación informática: servicio de mensajería por correo electrónico y SMS; circulares informativas por perfiles; firma electrónica de informes sociales. Para ello será necesario crear una vista de usuario en la aplicación actual para que el ciudadano pueda acceder, a través de su carpeta personalizada, a la situación de sus prestaciones; así como información sobre las mismas. En ella recibirá la información que su trabajador o trabajadora social considere de interés, según su caso y perfil.

2. Facilitar la interacción con el trabajador y la trabajadora social (profesional de referencia de la Atención Social Primaria), para conocer el estado de las prestaciones solicitadas.

3. Reducción de tiempos de tramitación de las ayudas y prestaciones sociales en un 50 por ciento: de 4-6 a 2-3 semanas en los casos de uso de las TIC una vez el proyecto esté totalmente desarrollado.

4. Sustitución de los contactos presenciales innecesarios por gestiones telemáticas al menos en un 50 por ciento en los casos de uso de las TIC, garantizando la atención presencial que el usuario precise.

5. Reducción de tiempos de espera en un 20 por ciento para ser atendido de manera presencial, en general, para todos/as los/las usuarios/as.

6. Difusión de la labor de los Servicios Sociales, así como de eventos y oportunidades, a través folletos informativos y redes sociales.

7. Creación de espacios de capacitación digital: aulas de informática e impartición de talleres de las TIC, con la optimización de las aulas y conexiones WIFI existentes en los 84 Centros Municipales de Mayores.

8. Uso de tablets en las visitas a domicilio: acceso a expedientes, aplicación de baremos en tiempo real, información in situ de frecuencia de los servicios, cálculo de copagos y valoración de necesidades en el propio entorno del usuario, mediante el uso de fotografías y apps útiles así como la posibilidad de firma del usuario para solicitud de prestaciones.

En la Figura 2 se resume el proyecto.

\subsection{Situación actual del proyecto}

En la actualidad (Enero de 2016), se ha finalizado ya una de las líneas del proyecto: todos los Centros de Servicios Sociales disponen de $t a-$ blets para sus trabajadores y trabajadoras sociales, que pueden emplearlas en las visitas domiciliarias además de para otros usos (reuniones,

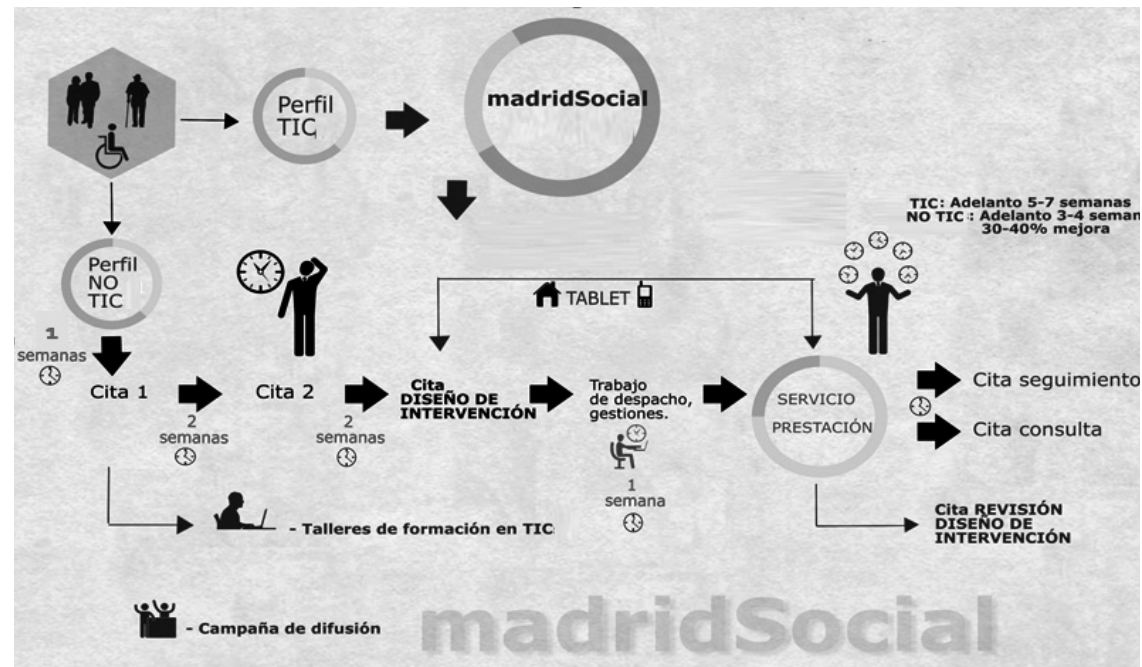

Figura 2. Esquema (blueprint) del proyecto.

Fuente: Elaboración propia. 
etc.), ya que tienen conexión $3 \mathrm{G}$ permanente. Se han entregado 4 tablets a cada uno de los 36 Centros de Servicios Sociales del municipio de Madrid.

Se están estudiando fórmulas para optimizar su uso y potenciar la visita a domicilio como técnica propia del Trabajo Social. Su realización es sinónimo de calidad en la atención que prestan los Servicios Sociales municipales, ya que a través de ella se pueden realizar diversas tareas en el propio entorno domiciliario (consultas de expedientes, baremos, registros, fotografía, citar, etc.).

Por otro lado, en estos momentos se está en espera de que se contraten y ejecuten los trabajos necesarios para modificar la herramienta informática actual de los Servicios Sociales municipales, con el fin de adaptarla a las nuevas funcionalidades requeridas por este proyecto, $\mathrm{y}$ que se prevé entren en funcionamiento a finales de 2016.

De la misma forma, durante 2016 se abordará lo relativo a la visibilización de los Servicios Sociales de Atención social primaria (ya se ha renovado la web municipal) por el Área de Gobierno de Equidad, Derechos Sociales y Empleo.

En fin, para llevar a cabo el seguimiento del proyecto, se ha constituido una comisión técnica formada por profesionales de los Servicios Sociales de varios distritos y por el Área de Gobierno. Con todo ello, esperamos que el presente proyecto pueda estar a pleno rendimiento en los Servicios Sociales de Atención primaria a lo largo de 2017.

\section{Conclusiones}

La Sociedad de la Información y el Conocimiento ha supuesto una revolución que no puede quedar al margen de un ámbito tan fundamental como el de los Servicios Sociales y la intervención social.

El potencial que encierran las TIC para la inclusión social exige que las instituciones implicadas en el bienestar de la ciudadanía se involucren en su utilización, ya que ofrecen posibilidades de comunicación, colaboración y eficiencia en la gestión hasta ahora impensables, y pueden multiplicar el alcance de las acciones de cualquier organización e iniciativa profesional.

Los Servicios Sociales municipales, como puerta de entrada al sistema público de Servicios Sociales, deben apropiarse de las TIC no sólo para la gestión interna, sino para la comunicación con la ciudadanía, dotándola de oportunidades para su inclusión social.

\section{Referencias bibliográficas}

Arias Astray, A., Alonso Gónzález, D. y Corchado Castillo, A.I. (2013). Trabajo Social en línea: Propuesta de una agenda de investigación para orientar su práctica. XII Congreso Estatal de Trabajo Social. Consejo General de Trabajo Social. Marbella, Noviembre.

Ayuntamiento de Madrid. (2015). Carta de Servicios de los Centros de Servicios Sociales de Atención Social Primaria. (Fecha de aprobación: 15 de julio de 2010. Fecha de la última evaluación: 23 de marzo de 2015). Carta Certificada 2014 N. recuperada de: http:/www.madrid.es/portales/ munimadrid/es/Inicio/El-Ayuntamiento/Servicios-sociales/Cartas-de-Servicios/Carta-de-Servicios-de-los-Centros-de-Servicios-Sociales-Atencion-Social-Primaria-Fecha-de-aprobacion-15de-julio-de-2010-Fecha-de-la-ultima-evaluacion-23-de-marzo-de-2015-Carta-Certificada-2014N?vgnextfmt $=$ default\&vgnextoid=0c707c2bbcaa8210VgnVCM2000000c205a0aRCRD\&vgnext channel=12a5ae1a5f2bb010VgnVCM100000d90ca8c0RCRD

Arredondo, R., Castellano, C. y Celimendiz, P. (2013). Intervención Social del S XXI. Congreso Estatal de Trabajo Social. Madrid: Consejo General de Trabajo Social de España. Marbella, Noviembre.

Arriazu R. y Fernández-Pacheco, J.L. (2013). Internet en el ámbito del Trabajo Social: formas emergentes de participación e intervención socio-comunitaria. Cuadernos de Trabajo Social, 26(1), $149-158$.

Colás, J. (2004). Las TIC en los Servicios Sociales. Red digital. Revista de Tecnologías de la Información y Comunicación Educativas, 5. Disponible en: http://reddigital.cnice.mecd.es/5/ firmas_nuevas/articulo7/colas_1.html (Consultado de 10/5/2015).

Cabrera, P.J., Rubio M.J., Fernández, Y., Fernández, S., Fernández, Y., Rúa, A., Fernández, E., López, J.A. y Malgesini, G. (2005). Nuevas Tecnologías y exclusión social. Un estudio sobre las posibilidades de las TIC en la lucha por la inclusión social en España. Madrid: Fundación Telefónica. 
Castells, M. (1997). La era de la información. Economía, sociedad y cultura. Volumen 1, La sociedad red. Alianza Editorial, Madrid.

Castells, M. (2007). Los mitos de Internet. Recuperado de: http://manuelgross.bligoo.com/content/ view/50516/Manuel-Castells-Los-mitos-de-Internet.html (consultado en enero de 2015)

Crovi, D. (2007). Acceso democrático, uso compartido y apropiación cultural de las TIC. Recuperado de: http://comunicacionyeducacionamic.blogspot.com.es/2007/07/accesodemocrtico-uso-compartido-y.html (Consultado en enero de 2015).

Federación Internacional de Trabajo Social. (IFSW) (2015). Definición de Trabajo Social aprobada por la Federación Internacional de Trabajo Social. Recuperado de: http://ifsw.org/propuesta-de-definicion-global-del-trabajo-social (Consultado en enero de 2015).

Fernández, T. y Ponce de León, L. (2014). Nociones Básicas de Trabajo Social. Madrid: Ediciones Académicas.

Fernández T. y Ponce de León, L (2016). Planificación y actuación estratégica de proyectos Sociales. Madrid: Ediciones Pirámide.

Gil Valenzuela, C. (1996). Sistema de Información de Usuarios de Servicios Sociales (SIUSS). Análisis crítico y alternativas. Documentos de Trabajo Social. Revista de Trabajo Social y Acción Social, 8, 90-195.

González Seara, L. (1981). Una democracia innovadora. Hemeroteca Digital El Pais. 24 de Mayo de 1981. Disponible en: http://elpais.com/diario/1981/05/24/opinion/359503205_850215.html

IMSERSO. (2011). Libro blanco sobre envejecimiento activo. Madrid: IMSERSO

Pérez, M., Sarasola, JL. y Balboa, M. (2012). Trabajo Social y Nuevas tecnologías. Portularia, XII. 57-60. Disponible en: http://www.redalyc.org/pdf/1610/161024437006.pdf (Consultado el 7/9/2015).

Puñal Romaris, M.E. (2004) Trabajo Social, Sistemas de Servicios Sociales y tecnológicas para la información: estudio cualitativo de la aplicación informática SIUSS. Portularia IV, 2004, 33-47. Disponible en: http://rabida.uhu.es/dspace/bitstream/handle/10272/254/b15139669.pdf?sequence $=1$, (Consultado el 10/01/2016).

Sociedad de la Información y el Conocimiento, (s. f). En Wikipedia. Recuperado de: http://es.wikipedia.org/wiki/Sociedad_de_la_informaci\%C3\%B3n. (Consultado el 10/04/2015). 
